\title{
Conversion Donut-Shaped Pattern to Directive Without Using Any Mode Converter
}

\author{
Seyed Jalil Hosseini \\ The School of Electrical Engineering, Iran University of Science and Technology, Tehran, Iran \\ Email address: \\ jalil_seyedhosseini@elec.iust.ac.ir \\ To cite this article: \\ Seyed Jalil Hosseini. Conversion Donut-Shaped Pattern to Directive Without Using Any Mode Converter. American Journal of \\ Electromagnetics and Applications. Vol. 9, No. 1, 2021, pp. 1-6. doi: 10.11648/j.ajea.20210901.11
}

Received: January 14, 2021; Accepted: March 18, 2021; Published: March 30, 2021

\begin{abstract}
In this paper, an electromagnetic diode with TEM mode, such as MILO, is connected directly to a Dual Mirrored Slotted Array Antenna (DMSAA). To convert a donut-shaped pattern of the electromagnetic source into a directive pattern, mode converters have been used in the past. But their losses, weight, size, and cost additions impact negatively on total system performance. Due to not using any mode converters, this is useful. Given the input is an electromagnetic diode with a TEM output mode, a large bandwidth is unnecessary. Therefore, slot antennas are used. Usually, array antennas were commonly used to increase the directivity and beamforming at the output of mode converters. To convert a donate-shaped pattern into a directive, half of the aperture fields must be rotated $180^{\circ}$. Here, the diode outlet is connected to the DMSAA with an inherent phase difference of $180^{\circ}$ of the electric fields. For this purpose, a power divider and a pair of narrow wall slotted array antennas (NWSAA) have been used. The antenna is simulated at $10 \mathrm{GHz}$ frequency. The results show a fully directive beam with a side lobe level (SLL) below $-10 \mathrm{~dB}$. Also, $\mathrm{S}_{11}$ is $-16.5 \mathrm{~dB}$, at $10 \mathrm{GHz}$ frequency. Because the mode converter is completely removed, this structure is very suitable for compact applications.
\end{abstract}

Keywords: Slotted Array Antenna, Power Divider, Mode Converter, Coaxial Waveguide, Rectangular Waveguide, Directive Antenna

\section{Introduction}

The output fields of electromagnetic diodes are mainly in TEM or $\mathrm{TM}_{01}$ mode [1-7]. If the electromagnetic field in the modes is propagated directly into space by a cone antenna or an open-ended waveguide, the radiation pattern will be donut-shaped [1-3]. Usually, mode converters are used to solve the problem. But, using the mode converters creates many problems, the most important of which are increasing the volume of the system, side lobe levels, and return losses [1, 3 , 8]. To increase the purity of the mode $\mathrm{TE}_{10}$ in the rectangular waveguide [2] (or $\mathrm{TE}_{11}$ in the circular waveguide [3]). Usually, after using a proper mode converter, the outlet is connected to an array antenna [9-13].

In this paper, we connect a coaxial waveguide directly to the slotted array antenna without any using mode converter, and a directive pattern is obtained.

In Section 2, we present the theory and design of the antenna. The section consists of three subsections. In Subsection 2.1, a power divider feeding two narrow-wall slotted array antenna is presented and design. In subsection 2.2 a DMSAA is introduced and design. Finally, the total structure is assembled and its results is presented. At the end, Conclusion is presented.

\section{Theory and Design}

If we consider the outlet of the electromagnetic diode as a coaxial waveguide, the electrical fields are arranged radially $[1,2]$. Assume, the coaxial waveguide consists of two 180-degree sectorial waveguides, so their electrical fields have a $180^{\circ}$ phase difference. The electrical fields in these two sectorial waveguides are in $\mathrm{TE}_{11}$ mode. Now, if these are connected to two rectangular waveguides, $\mathrm{TE}_{10}$ mode is excited in these [13]. The electrical fields in the rectangular waveguides have a $180^{\circ}$ phase difference. Now, if the waveguides are connected to the inlets of two slot array antennas where the slots are symmetrical. It is like where two similar slot array antennas are fed by two fields which have a $180^{\circ}$ phase difference. The design of the system consists of 
two main parts. A $1 \times 2$ coaxial to rectangular power divider, and a DSMAA.

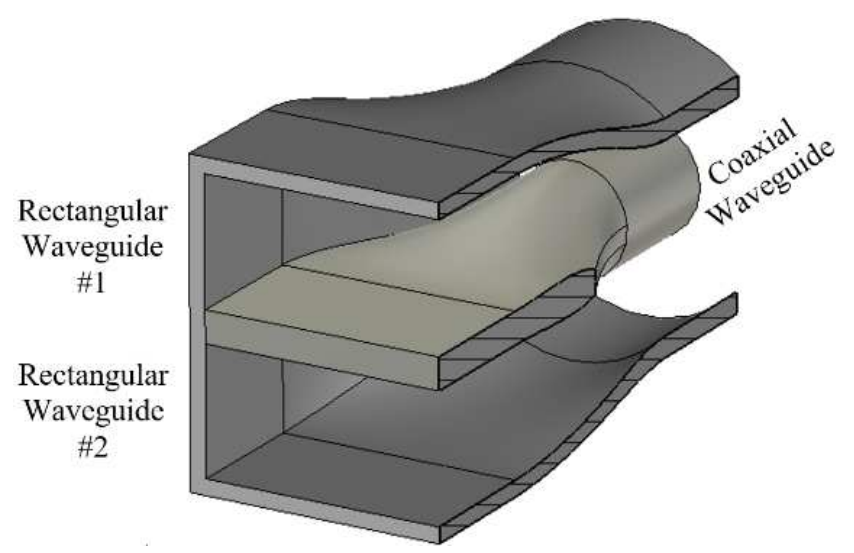

Figure 1. $1 \times 2$ coaxial to rectangular power divider.

\section{1. $1 \times 2$ Coaxial to Rectangular Power Divider}

All of the antennas are usually fed by special structures [14], but here the simple structure is used. A coaxial waveguide consists of a body and core [15]. In this paper, the circular body of the coaxial waveguide is transformed into a

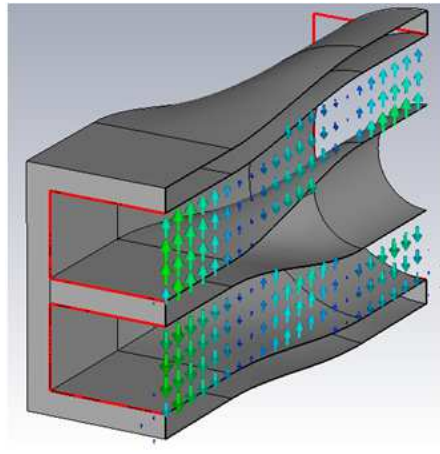

a

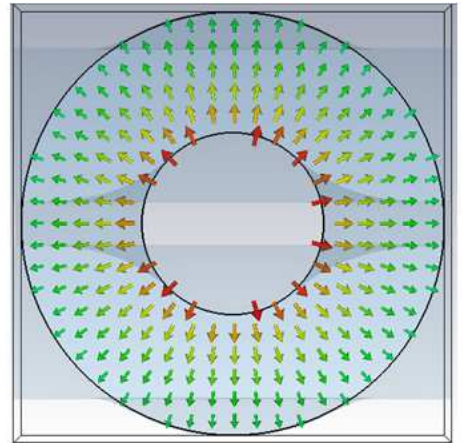

b rectangular waveguide, with small changes. The coaxial waveguide core also slowly turns into a plate. In Figure 1, the $1 \times 2$ coaxial to rectangular power divider is shown.

Since this system must be designed at $10 \mathrm{GHz}$ frequency, to reduce the possibility of excitation of higher modes, the width of the rectangular waveguides must be selected appropriately. So, we have [14]:

$$
\begin{aligned}
f_{c_{(T E 10)}} & =\frac{c}{2 a} \\
f_{c_{(T E 20)}} & =\frac{c}{a}
\end{aligned}
$$

where, $f_{c_{(T E 10)}}$ and $f_{c_{(T E 20)}}$ are cut-off frequencies of the dominant and second modes excited in an air-filled rectangular waveguide, respectively. Also, $c$ and $a$ are lightspeed and the rectangular waveguide width, respectively. Now, if it is assumed $b>a / 2$, the second mode excited in the rectangular waveguide is $\mathrm{TE}_{20}(b$ is the height of the outlet waveguides). The inner diameter of the coaxial waveguide body is approximately $20 \mathrm{~mm}$. For the impedance of the coaxial waveguide to be equal to $50 \Omega$, the core diameter must be equal to 0.43 of the body. Figure 2 shows the electric fields at the input and output of the power divider.

Figure 2. The electrical fields along with $1 \times 2$ coaxial to rectangular power divider, a) Longitudinal cut-view, b) coaxial waveguide input, and c) rectangular waveguide output.

If the input, and upper and downer output ports are numbered 1, 2, and 3, respectively, the S parameters are as Figure 3.

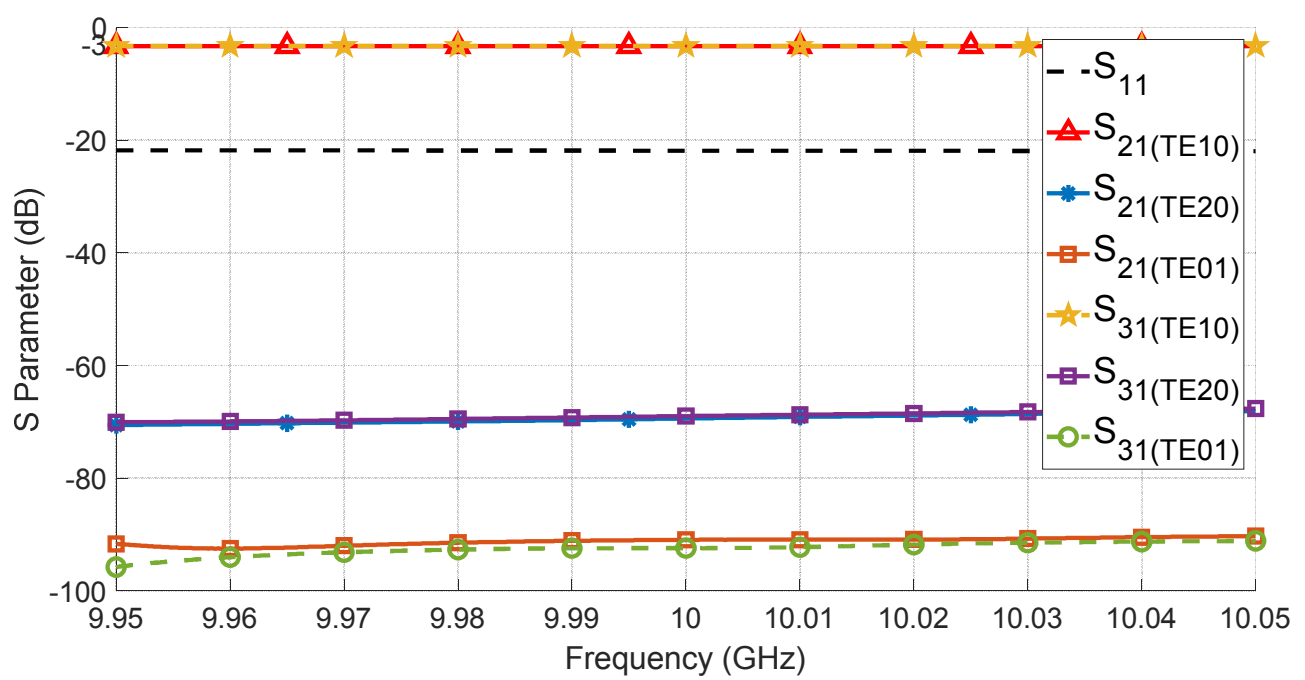

Figure 3. $S$ parameters $1 \times 2$ coaxial to rectangular power divider. 


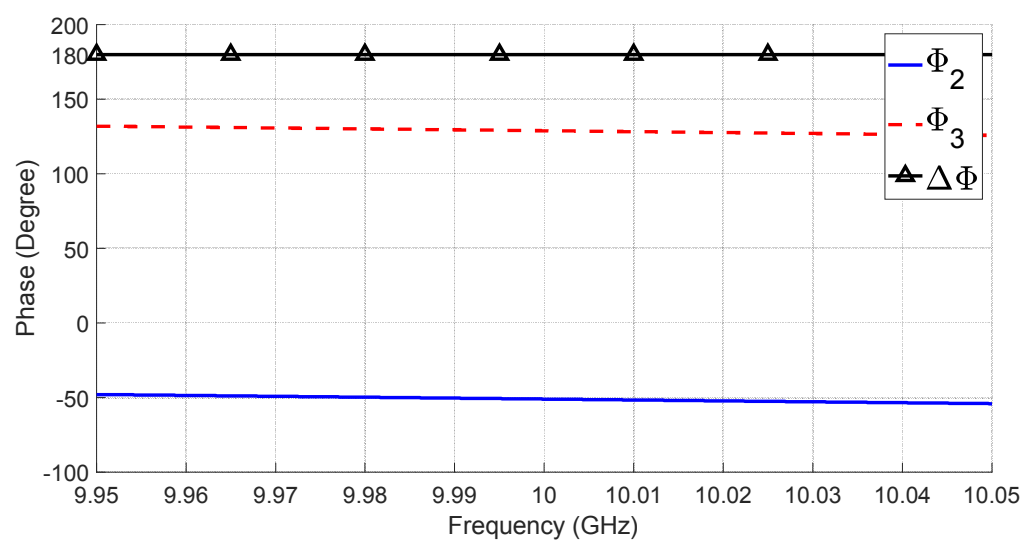

Figure 4. Output port phases and their difference.

According to Figure 3, the electrical field strength at the outputs is equal in ports 2 and 3 . Also, the strengths of the higher modes are very weak in both output ports. Furthermore, the $\mathrm{S}_{11}$ is less than $-20 \mathrm{~dB}$, which is very good. According to the theory described above, the electric fields in the two output rectangular waveguides have a phase difference of $180^{\circ}$. This is shown in Figure 4.

\subsection{Antenna Design}

In this paper, an NWSAA is used. Next, an NWSAA is designed, and another is completely symmetrical. To design an NWSAA, first, the width, height, and thickness of the waveguide are selected. In this design, the widths of all slots are equal, and they are selected one-twentieth of the free-space wavelength [15]. To design an NWSAA, the following must be done in order [16-18]:

1. Calculate the free space wavelength as follows,

$$
\lambda_{0}=\frac{c}{f}
$$

2. Calculate the width of the slots as follows,

$$
W_{\mathrm{s}}=\frac{\lambda_{0}}{20}
$$

3. Selecting the width, height, and thickness of the waveguide, Calculating the guide wavelength as follows,

$$
\lambda_{\mathrm{g}}=\frac{\lambda_{0}}{\sqrt{1-\left(\frac{\lambda_{0}}{2 a}\right)^{2}}}
$$

4. Calculating the distance of the slot centers as follows,

$$
S_{\mathrm{s}}=\frac{\lambda_{\mathrm{g}}}{20}
$$

5. Calculating the amplitude coefficient of each slot. These parameters are calculated by Array Factor. The radiation pattern is actually a multiplication of the element's radiation pattern or called Array Factor. Array Factor can be expressed by the following formula,

$$
\begin{gathered}
F_{2 M}=\sum_{n=1}^{M} a_{n} \cos [(2 n-1) \psi] \\
\psi=\frac{\pi S_{S}}{\lambda_{0}} \cos \varphi \\
\bar{E}_{\text {total }}=\bar{E}_{\text {element }} \times F
\end{gathered}
$$

Not, Array Factor of a uniform array is as

$$
a_{n}=\frac{\sin \left(\frac{n \psi}{2}\right)}{N \sin \left(\frac{\psi}{2}\right)}
$$

6. Calculating the normalized resonant conductance of the slots as follows,

$$
g_{n}=\frac{a_{n}^{2}}{\sum_{i=1}^{N} a_{i}^{2}} ; i=1,2, \ldots, N
$$

7. Calculating the angle between the axis elements and the vertical as follows,

$$
\theta_{n}=-900.7 g_{n}^{2}+237.6 g_{n}+2
$$

8. Calculating the depth of slots as follows,

$$
d_{n}=t+\left[0.4625 \lambda_{0}-\left(\frac{\frac{b}{\cos \left(\frac{\theta_{n}}{180}\right)}}{2}\right)\right]
$$

By the aforesaid nine steps, NWSAA can be designed. The dimensions derived from these steps, at $10 \mathrm{GHz}$ frequency, are given in Figure 5 and Table 1.

Table 1. The values of the parameters.

\begin{tabular}{llll}
\hline Parameter & $\begin{array}{l}\text { Value (mm or } \\
\text { degree) }\end{array}$ & Parameter & $\begin{array}{l}\text { Value (mm \& } \\
\text { degree) }\end{array}$ \\
\hline $\mathrm{a}$ & 21.4 & $\theta_{6}$ & 19.33 \\
$\mathrm{~b}$ & 9.5 & $\theta_{7}$ & 17.90 \\
$\mathrm{t}$ & 1.2 & $\theta_{8}$ & 15.14 \\
$\mathrm{Ro}$ & 20.6 & $\theta_{9}$ & 11.99 \\
$\mathrm{Ri}$ & 8.6 & $\theta_{10}$ & 13.35 \\
$\mathrm{Ws}$ & 1.5 & $\mathrm{~d}_{1}$ & 2.05 \\
$\mathrm{Ss}$ & 21.0 & $\mathrm{~d}_{2}$ & 2.06 \\
$\mathrm{Lw}$ & 19.4 & $\mathrm{~d}_{3}$ & 1.99 \\
$\mathrm{Lt}$ & 9.6 & $\mathrm{~d}_{4}$ & 1.91 \\
$\mathrm{Lm}$ & 20.8 & $\mathrm{~d}_{5}$ & 1.87 \\
$\theta_{1}$ & 12.86 & $\mathrm{~d}_{6}$ & 1.87 \\
$\theta_{2}$ & 11.66 & $\mathrm{~d}_{7}$ & 1.92 \\
$\theta_{3}$ & 15.16 & $\mathrm{~d}_{8}$ & 1.99 \\
$\theta_{4}$ & 19.13 & $\mathrm{~d}_{9}$ & 2.06 \\
$\theta_{5}$ & 20.88 & $\mathrm{~d}_{10}$ & 2.04 \\
\hline
\end{tabular}

\subsection{Total System}

Figure 6 shows the final structure made by connecting a $1 * 2$ coaxial power divider to a DMSAA. 


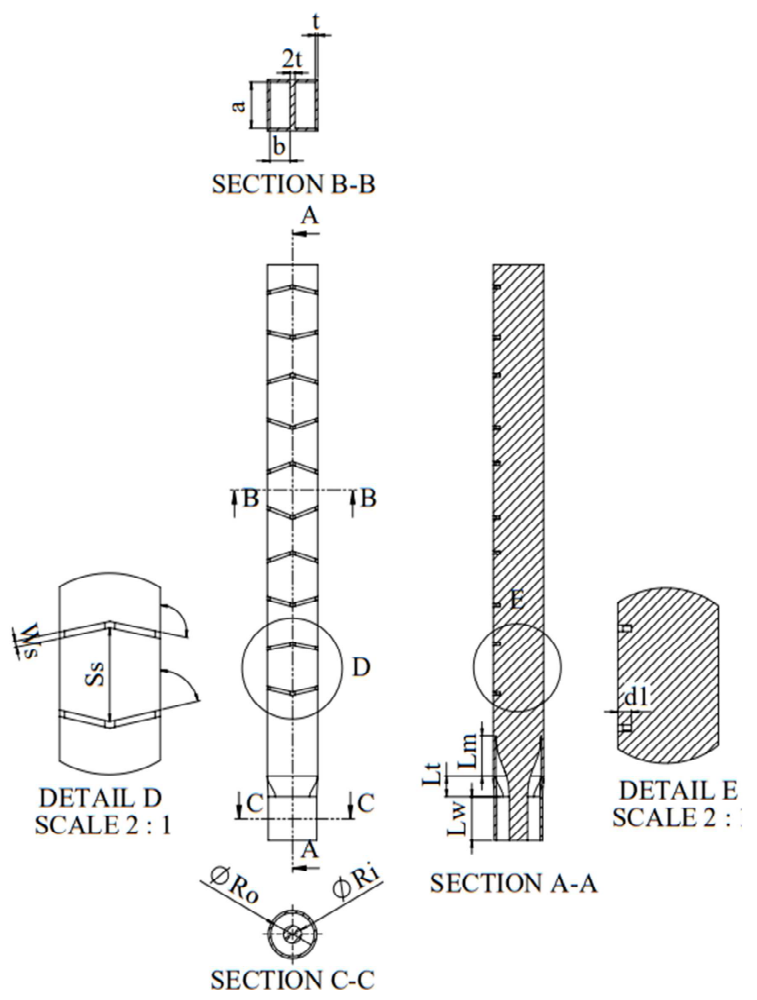

Figure 5. The parameters of the total system.

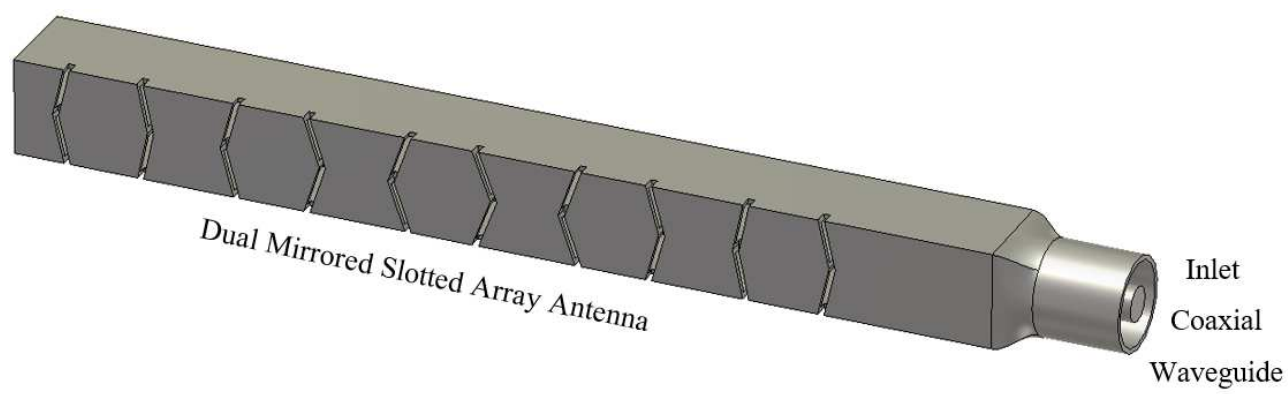

Figure 6. An overview of the total system.

In Figure 7, its radiation patterns are shown.

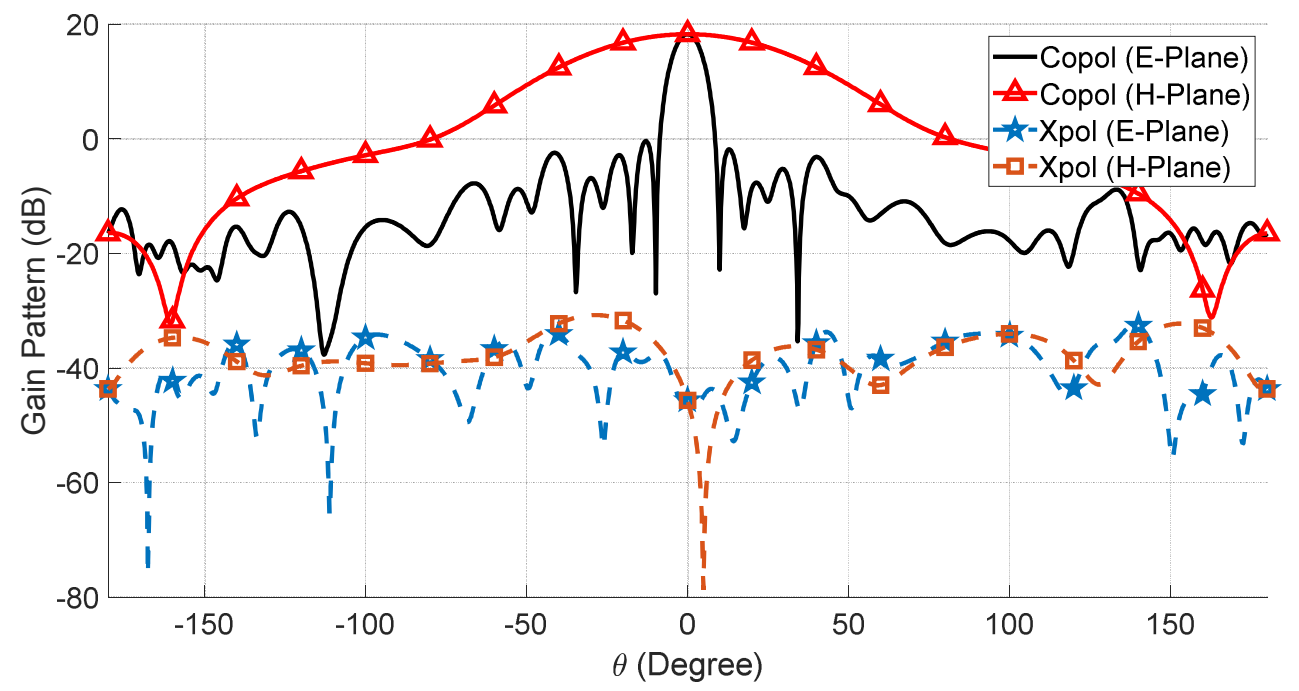

Figure 7. The radiation gain patterns of the DMSAA, in the E and H plates. 
According to the antenna patterns, it is clear that at $\theta=0$, the antenna patterns, despite the coaxial inlet, are completely directive in both plates. In Figure 8, the total $\mathrm{S}_{11}$ is shown.

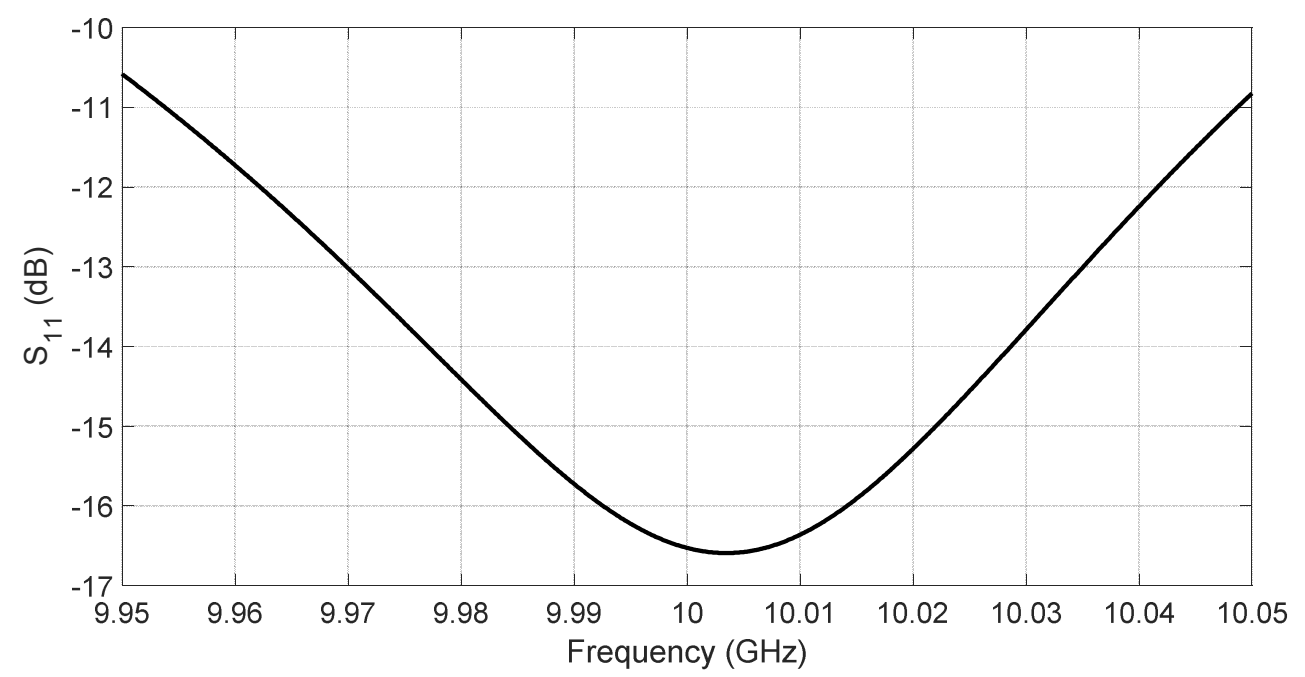

Figure 8. total $S_{11}$.

\section{Conclusion}

We can conclude that without the use of a mode converter, a direct radiation pattern can be obtained. If a DMSAA is used, the $180^{\circ}$ phase difference between the upper and lower waveguides can be removed. In this article, the topic is designed and simulated. The total $\mathrm{S}_{11}$ is $-16.6 \mathrm{~dB}$, at the 10 $\mathrm{GHz}$ frequency. Also, the SLL is $-20 \mathrm{~dB}$ and $\mathrm{x}-$ pol radiation patterns are less than $-30 \mathrm{~dB}$. Due to non-use any mode converter, the cost and volume of the total structure is decrease, dramatically.

\section{References}

[1] S. J. Hosseini, R.-A. Sadeghzadeh, and H. Aliakbarian, “A TEM-TE11 mode-transducing sectoral antenna by using dual dielectric window," International Journal of Electronics Letters, vol. 6, no. 4, pp. 403-412, Oct. 2018, doi: $10.1080 / 21681724.2017 .1381997$.

[2] S. J. Hosseini, M. Dahmardeh, and M. Yousefian, "A high power TEM to TE10 mode converter with $70 \%$ bandwidth," Journal of Electromagnetic Waves and Applications, vol. 0, no. 0, pp. 1-11, Nov. 2020, doi: 10.1080/09205071.2020.1842255.

[3] S. J. Hosseini and H. Oraizi, "TEM-TE11 mode converter antenna like a pelican beak," IET Microwaves, Antennas \&amp; Propagation, vol. 14, no. 9, pp. 934-942, Apr. 2020, doi: 10.1049/iet-map.2020.0017.

[4] X.-Y. Wang, Y.-W. Fan, T. Shu, C. Yuan, and Q. Zhang, “A high-efficiency tunable TEM-TE11 mode converter for high-power microwave applications," AIP Advances, vol. 7, no. 3, p. 035012, Mar. 2017, doi: 10.1063/1.4978863.

[5] A. Chittora, S. Singh, A. Sharma, and J. Mukherjee, "A Novel \$ \rm TM01 to TE11 Mode Converter Designed With Radially Loaded Dielectric Slabs," IEEE Transactions on Microwave Theory and Techniques, vol. 64, no. 4, pp. 1170-1175, Apr. 2016, doi: 10.1109/TMTT.2016.2536031.
[6] X. Li, X.-Q. Li, Q.-X. Liu, and J.-Q. Zhang, "HIGH POWER OVER-MODE BENT WAVEGUIDES FOR CIRCULAR TM01 AND COAXIAL TEM MODE TRANSMISSION," Progress In Electromagnetics Research M, vol. 60, pp. 189196, Jan. 2017, doi: 10.2528/PIERM17080901.

[7] S. J. Hosseini, and M. Dahmardeh, "Compact smile-like mode converter antenna with high power capacity level," Electromagnetics, pp. 1-17, Mar. 2021, doi: $10.1080 / 02726343.2021 .1903216$.

[8] M. M. Honari, R. Mirzavand, S. Aslanzadeh, H. Saghlatoon, and P. Mousavi, "Wideband Printed TM01 to TE11 Mode Converters," IEEE Access, vol. 7, pp. 35438-35448, 2019, doi: 10.1109/ACCESS.2019.2904247.

[9] J. W. Li, G. J. Deng, L. T. Guo, W. H. Huang, and H. Shao, "Polarization controllable TM01-TE11 mode converter for high power microwaves," AIP Advances, vol. 8, no. 5, p. 055230, May 2018, doi: 10.1063/1.5026962.

[10] X. Zhao, C. Yuan, L. Liu, S. Peng, H. Zhou, and D. Cai, "Solution to GW TEM-Circular Polarized TE11 Mode Converter Design for High Frequency Bands," IEEE Transactions on Microwave Theory and Techniques, vol. 65, no. 2, pp. 432-437, Feb. 2017, doi: 10.1109/TMTT.2016.2616880.

[11] Y. Sydorenko, S. Provalov, S. Shylo, and D. Wheeler, "Compact MMW-band Planar Diffraction Type Antennas for Various Applications," American Journal of Electromagnetics and Applications, vol. 8, no. 1, Art. no. 1, Feb. 2020, doi: 10.11648/j.ajea.20200801.13.

[12] A. Chittora, S. Singh, A. Sharma, and J. Mukherjee, "A TM01 to TE11 mode converter designed with semicircular waveguide sections," in 2017 11th European Conference on Antennas and Propagation (EUCAP), Mar. 2017, pp. 19031906, doi: 10.23919/EuCAP.2017.7928152.

[13] L. Yu, C. Yuan, J. He, and Q. Zhang, "Beam Steerable Array Antenna Based on Rectangular Waveguide for High-Power Microwave Applications," IEEE Transactions on Plasma Science, vol. 47, no. 1, pp. 535-541, Jan. 2019, doi: 10.1109/TPS.2018.2884290. 
[14] B. Bandyopadhyay, S. Chatterjee, A. Majumder, and S. Chatterjee, "Narrow Wall Slotted Planar Array Antenna by using Differential Feed at X Band," in 2019 IEEE Asia-Pacific Microwave Conference (APMC), Dec. 2019, pp. 521-523, doi: 10.1109/APMC46564.2019.9038641.

[15] M. Yousefian, S. J. Hosseini, and M. Dahmardeh, "Compact broadband coaxial to rectangular waveguide transition," Journal of Electromagnetic Waves and Applications, vol. 33, no. 9, pp. 1239-1247, Jun. 2019, doi: $10.1080 / 09205071.2019 .1606737$.

[16] Moh. A. K. S. Lubis, C. Apriono, F. Y. Zulkifli, and E. T. Rahardjo, "Design of narrow wall slotted waveguide antenna for X-band application," in 2017 Progress in Electromagnetics
Research Symposium - Fall (PIERS - FALL), Nov. 2017, pp. 2625-2628, doi: 10.1109/PIERS-FALL.2017.8293579.

[17] M. Dolati and M. S. Majedi, "A wideband $45^{\circ}$ inclined linear polarization travelling-wave slot array antenna with broadside radiation pattern," AEU - International Journal of Electronics and Communications, vol. 106, pp. 103-107, Jul. 2019, doi: 10.1016/j.aeue.2019.05.007.

[18] Y. Y. Sun, S. W. Cheung, and T. I. Yuk, "An ISM/UWB Antenna with Offset feeding and Slotted Ground Plane for Body-centric Communications," Journal of Electrical and Electronic Engineering, vol. 1, no. 2, Art. no. 2, Jan. 2014, doi: 10.11648/j.jeee.20130102.12. 